\title{
Intraoperative Bildgebung in der Mund-, Kiefer- und Gesichtschirurgie
}

\author{
Henning Hanken, Christian Lohse, Alexandre T. Assaf, Max Heiland
}

\section{Zusammenfassung}

Der Gesichtsschädel ist in der heutigen Zeit häufig von Verletzungen betroffen. Neben körperlichen Auseinandersetzungen, Fahrradstürzen und Sportunfällen führen Schussverletzungen zu unterschiedlichsten Verletzungsmustern, die ästhetische, aber auch funktionelle Defizite bei den Betroffenen hinterlassen können. In diesem Zusammenhang ist die korrekte 3-dimensionale Rekonstruktion des Gesichtsschädels von entscheidender Bedeutung. Heute verfügbare intraoperative bildgebende Verfahren sind in der Lage, die Reposition und die Rekonstruktion des Gesichtsschädels mehrdimensional zu visualisieren, um ggf. eine Revision sofort durchführen $\mathrm{zu}$ können. Die komplexe 3-dimensionale Struktur des Gesichtsschädels stellt dabei besondere Anforderungen an die intraoperativen bildgebenden Verfahren, da nur eine 3-dimensionale Bildgebung eine ausreichende Beurteilung des Operationserfolgs zulässt. Dieser Übersichtsartikel beschreibt die aktuellen intraoperativen bildgebenden Verfahren mit Sonografie, CT, MRT und DVT einschließlich ihrer Vor- und Nachteile aus der Sicht der Mund-, Kiefer- und Gesichtschirurgie.

\section{Intraoperative Imaging of the Facial Skeleton}

The head and neck are often affected by various causes of injuries. In addition to physical confrontations, bicycle crashes and accidents, gunshot wounds cause injury patterns which can lead to aesthetic and also functional deficits. In this context, a correct three-dimensional reconstruction of the facial skeleton is of crucial importance. The complex three-dimensional structure of the head and neck is demanding for intraoperative imaging modalities. Only a three-dimensional imaging technique allows an adequate assessment of the success of the operation in this field. Nowadays, intraoperative imaging techniques allow an immediate examination of the reduction and reconstruction and enable an immediate revision, if needed. This review article describes the current intraoperative imaging modalities including ultrasound, CT, MRI, and CBCT as well as their main disadvantages and advantages from the point of view of oral and maxillofacial surgery.

\section{Einführung}

Der Gesichtsschädel ist in allen Altersgruppen häufig von Verletzungen betroffen. Die komplexe Anatomie des Gesichtsschädels stellt nach einem Trauma höchste Anforderungen an die bildgebenden Verfahren zur Diagnostik sowie in der Therapieplanung und -durchfüh-

OP-JOURNAL 2013; 29: 130-135

(c) Georg Thieme Verlag KG Stuttgart · New York DOI http://dx.doi.org/10.1055/s-0033-1350665

rung. Neben ästhetischen können insbesondere funktionelle Defizite, zum einen durch das Trauma, zum anderen durch den chirurgischen Eingriff, entstehen. Die operative Therapie kann in $3 \mathrm{Ab}$ schnitte gegliedert werden:

1. Diagnostik mit Stellen der Indikation zur operativen Versorgung, Auswahl der benötigten Implantate, Festlegung der Zugangswege und ggf. Vorbereitung zur intraoperativen Navigation

2. Operation

3. Reevaluation nach dem Eingriff - die zweckmäßigerweise unmittelbar nach
Versorgung noch in Narkose erfolgen sollte, sodass Korrekturen direkt durchgeführt werden können.

Hier nimmt die intraoperative Bildgebung eine wichtige Stellung im Hinblick auf den Erfolg des chirurgischen Eingriffs ein. Die heute zur Verfügung stehenden und zum Einsatz kommenden Bildgebungsverfahren beruhen auf meist modifizierten, etablierten Röntgen- oder Schnittbildverfahren wie C-Bogen/DVT (digitale Volumentomografie), Computer- (CT) oder auch Magnetresonanztomografien (MRT). Aber auch Ultraschalluntersuchungen (US) stellen eine Möglichkeit zur intraoperativen Bildgebung dar. Sie alle sollen den Erfolg des chirurgischen Eingriffs erhöhen und eine mögliche Sekundäroperation vermeiden. Im optimalen Fall soll eine interventionsbedürftige Fehlstellung nach Versorgung, suboptimale Positionierung der Implantate und eine mögliche Verletzung relevanter Strukturen durch fehlplatzierte Schrauben noch in der Erstoperation erkannt werden und dies die Möglichkeit einer sofortigen Revision eröffnen.

Die intraoperative Bildgebung erhöht den Erfolg eines chirurgischen Eingriffs und hilft, Sekundäroperationen zu vermeiden.

Ferner entstand der Wunsch, die bereits präoperativ in der Diagnostik erhobenen Daten für den intraoperativen Einsatz zur besseren Orientierung nutzbar zu machen. Hier kann die computergestützte/computerassistierte Chirurgie den Operateur bei der Wiederherstellung der korrekten Struktur in Form und Größe unterstützen.

\section{Das optimale intraoperative Bildgebungssystem}

Die Anforderungen an ein optimales intraoperatives Bildgebungsverfahren können je nach Untersuchung abweichen. 


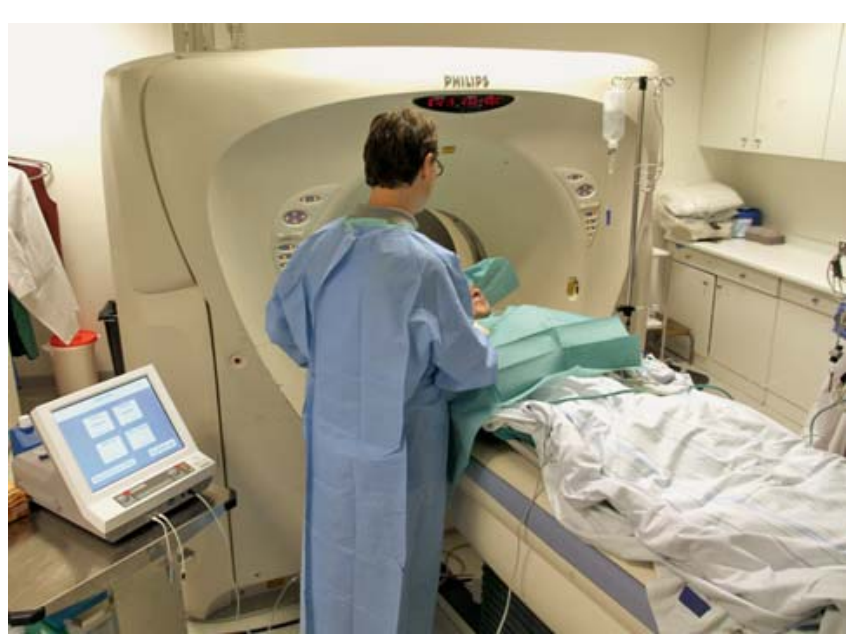

Abb. 1 Ein intraoperatives stationäres CT-Gerät, das unter sterilen Bedingungen eingesetzt werden kann. Exemplarisch ist hier eine CT-gesteuerte Ablation eines Hirnnervs zu sehen.
Doch im Allgemeinen lässt sich folgendes festhalten:

Unabdingbar für den Patienten und das chirurgische Personal sind gewisse Sicherheitsaspekte. Für das Personal, falls es im Operationssaal bleiben soll, ist die Nutzung eines bildgebenden Verfahrens ohne ionisierende Strahlung vorteilhaft. Falls dennoch Röntgenstrahlung zum Einsatz kommt, ist unbedingt auf einen genügenden Sicherheitsabstand zum Gerät und auf das Tragen von Bleischürzen zu achten. Darüber hinaus sollte das genutzte bildgebende Verfahren jederzeit die Überwachung der lebensnotwendigen Körperfunktionen und auch einen freien Patientenzugang ermöglichen bzw. sollte diesen nicht einschränken. Außerdem sollte der Operationsablauf durch die bildgebende Diagnostik möglichst nicht gestört bzw. verzögert werden.

Das optimale intraoperative Bildgebungssystem liefert zudem schnell (in möglichst kurzer Zeit der Durchführung) hochauflösende 3-dimensionale Bilder in einem ausreichend groß erfassten Bildausschnitt (,field of view“) mit nur gering ausgeprägten Metallartefakten. Dabei sollte die Strahlenbelastung für den Patienten niedriger als bei der sonst verwendeten prä- und postoperativen Diagnostik sein.

\section{Intraoperative Bildgebungsverfahren}

\section{Ultraschall (US), Sonografie}

Der Schall ist eine Materiewelle, bei der die Moleküle oder Atome der Materie in Richtung der Schallausbreitung schwingen. Beim Übergang von Materie unterschiedlicher Dichte ändert sich die
Schallgeschwindigkeit aufgrund der Wellenbrechung, und ein Teil der Welle wird an der Übergangsfläche gebrochen. Der Ultraschall hat eine Frequenz von mehr als $16000 \mathrm{~Hz}$ und ist für das menschliche Ohr nicht hörbar. In der medizinischen Diagnostik werden Frequenzen zwischen 2 und $20 \mathrm{MHz}$ verwendet. Er wird durch elektrische Kristalle erzeugt, die beim Anlegen einer entsprechenden elektrischen Wechselspannung im Rhythmus der Spannung schwingen und den Ultraschall erzeugen. Im Prinzip wird ein Ultraschallbild in der Medizin dadurch erzeugt, dass der in den Körper eingestrahlte Ultraschall an den verschiedenen Geweben unterschiedlich stark reflektiert wird. Ultraschalluntersuchungen von luftgefüllten Organen wie Lunge oder MagenDarm-Trakt sind nicht möglich, genauso sind Untersuchungen ab einer bestimmten Eindringtiefe Grenzen gesetzt. Zudem kann man mittels US die Fließgeschwindigkeit des Blutes (Doppler-Sonografie) beurteilen. Das Potenzial von intraoperativen Ultraschalluntersuchungen für die Mund-, Kiefer- und Gesichtschirurgie liegt v.a. in der Visualisierung der Jochbögen sowie der Vorderwand der Stirnhöhle. Ein großer Vorteil ist die Möglichkeit der intraoperativen Darstellung nach geschlossener Reposition, ohne den Patienten einer zusätzlichen Strahlenbelastung auszusetzen [1,2]. Zudem zeigt die Sonografie eine gute Weichgewebsdarstellung, was intraoperativ zur Lokalisation von Lymphknoten, Abszesshöhlen u.a. genutzt werden kann. Im Vergleich zu den alternativ zur Verfügung stehenden Bildgebungsverfahren wie CT oder MRT geht die Ultraschalldiagnostik nicht mit einem hohen personellen und apparativen Aufwand einher. Durch die Kopplung der Bild- daten mit modernen Navigationssystemen ist es so zudem möglich, die Ultraschalldiagnostik in der computergestützten, computerassistierten Chirurgie zum Einsatz zu bringen, was bspw. in der Neurochirurgie zur Korrektur des Brain Shifts nach Eröffnen der Kalotte genutzt wird. Nachteile der Sonografie bestehen in der geringen Eindringtiefe, die durch Knochen limitiert wird, sowie der begrenzten 3-dimensionalen Darstellbarkeit insbesondere von Knochenstrukturen. Zur Darstellung und Beurteilung von komplexen und ausgedehnten Verletzungen des Gesichtsschädels ist die Sonografie nicht geeignet.

Intraoperative Ultraschalluntersuchungen liefern schnell und ohne Strahlenbelastung Informationen über das Weichgewebe und in sehr begrenztem Maße über oberflächliche Knochenstrukturen. Für die Beurteilung von ausgedehnten Verletzungen des Gesichtsschädels ist die Nutzung des Ultraschalls aber nicht geeignet.

\section{Computertomografie (CT)}

Die Computertomografie ist eine Technologie, die nicht mehr aus der modernen bildgebenden Diagnostik wegzudenken ist. Für den Operationssaal wurde diese Technik modifiziert hin zu kleineren Geräten, sodass eine Integration in den Operationsaal und Operationsablauf möglich wurde [3].

In diesem Zusammenhang wurde auch der Begriff des „Hybrid-Operationssaals“ eingeführt, da ein Saal mit einem stationären CT-System sowohl für eine Operation als auch für die Bildgebung genutzt werden kann. Diesem räumlichen Konzept steht eine Alternativlösung entgegen, bei der das CT-Gerät mobil in mehreren Operationssälen eingesetzt werden kann, indem es mit einem ausklappbaren Schienungssystem bei Bedarf fest an entsprechende Operationstische gekoppelt werden kann. Abb. 1 zeigt ein Beispiel für einen typischen Hybrid-Operationssaal mit einem stationären CT-Gerät.

Die Vorteile der Computertomografie für die intraoperative Bildgebung liegen in der Güte und Qualität des Bildmaterials, das in der Regel hochauflösend Weichund Hartgewebe wiedergibt. Die Nachteile der intraoperativen CT-Bildgebung liegen in den Kosten für das Gerät, der Strahlenbelastung für den Patienten, die als insgesamt hoch einzustufen ist, in der 


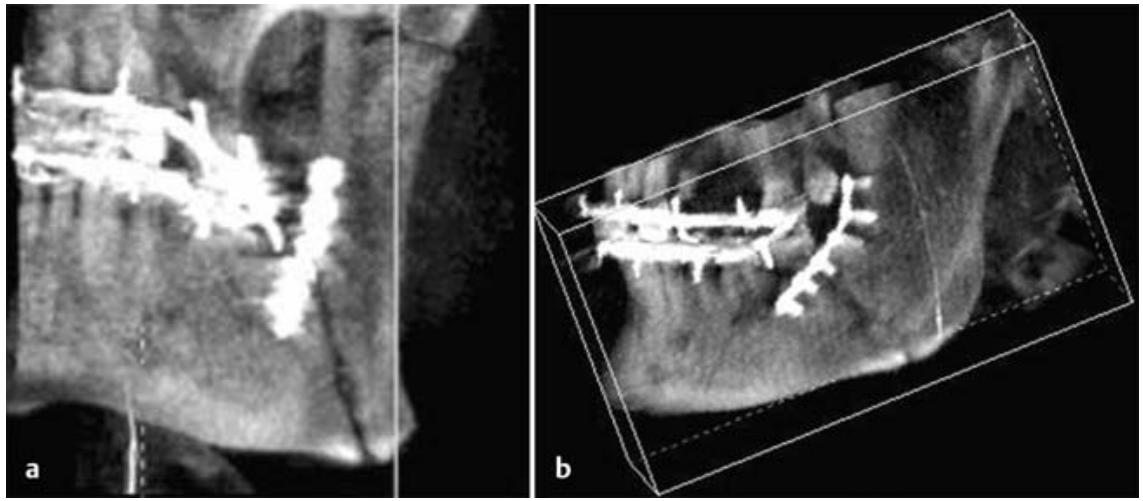

Abb. $\mathbf{2 a}$ und $\mathbf{b}$ Intraoperative Darstellung einer osteosynthetisch versorgten Kieferwinkelfraktur links mit einem 3-D-C-Bogensystem (Siemens ARCADIS Orbic 3D, Siemens, Erlangen, Deutschland). Die Fraktur zeigt sich adäquat reponiert. Obwohl im Ober- und Unterkiefer eine Drahtbogenkunststoffschiene nach Schuchardt eingebracht wurde, sind die Metallartefakte in der 3-dimensionalen Rekonstruktion vernachlässigbar.
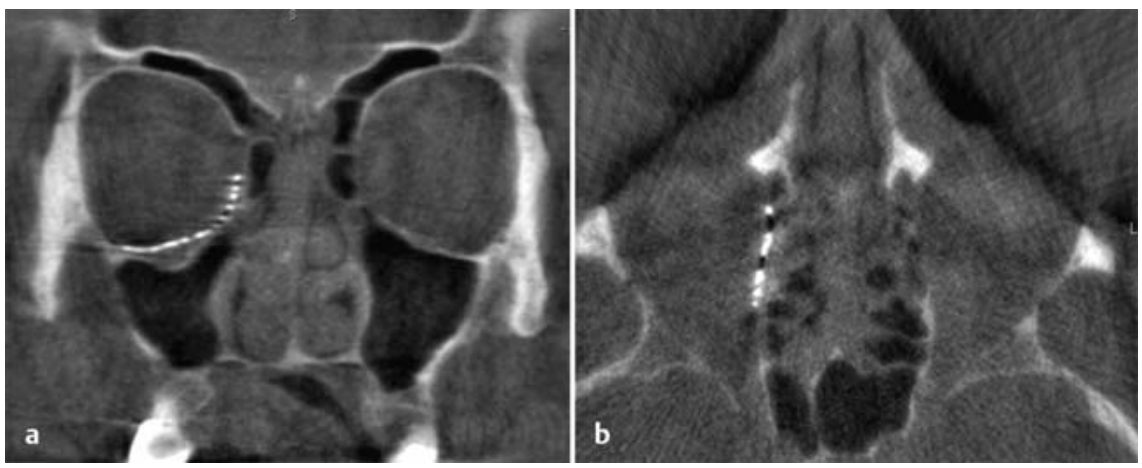

Abb. $\mathbf{3}$ a und b Intraoperative Darstellung eines durch ein Titanmesh (MatrixMIDFACE Preformed Orbita Plate, DePuy Synthes $\mathrm{GmbH}$, Umkirch) rekonstruierten Orbitabodens rechts bei großflächiger Fraktur mit einem 3-D-C-Bogensystem (Siemens ARCADIS Orbic 3D, Siemens, Erlangen). Der Boden und die mediale Wand zeigen sich in den Ebenen adäquat rekonstruiert.

Notwendigkeit radiologischen Fachpersonals und im intraoperativen Zeitverlust, der aufgrund der Durchführung der Aufnahme entsteht.

Die intraoperative CT-Bildgebung liefert hochqualitative 3-dimensionale Bilder vom Weich- und Hartgewebe. Allerdings sind die CT-Geräte kostspielig, und der Patient wird einer relativ hohen Strahlenbelastung ausgesetzt.

\section{Magnetresonanztomografie (MRT)}

Im Gegensatz zur konventionellen Röntgendiagnostik, der Computertomografie (CT) oder der digitalen Volumentomografie (DVT) wird bei der Durchführung der Magnetresonanztomografie (MRT) keine ionisierende Strahlung erzeugt. Moderne MRT-Geräte arbeiten mit starken statischen Magnetfeldern oder magnetischen Wechselfeldern. Die Aktivierung dieser magnetischen Felder führt zu einer phasensynchronen Anregung bestimmter Atomkerne (Wasserstoffkerne/Protonen), die dann wiederum eine Wechselspannung abgeben und durch die Auslenkung der Atomkerne ein elektromagnetisches Signal erzeugen. Beim Abschalten dieses HF-Magnetfelds geben sie magnetische Signale ab, die von Empfangsspulen empfangen werden. Über zeitweilig zugeschaltete GradientenMagnetfelder können diese Signale bestimmten Orten und präzisen Volumenelementen beim Patienten zugeordnet werden.

Heutige MRT-Geräte in der klinischen Anwendung arbeiten in der Regel im 1,0-1,5-Tesla-(T-)Bereich (Niederfeldgeräte) oder 3-T-Bereich (Hochfeldgeräte) und erzeugen ebenso wie CT- oder DVTGeräte Schnittbilder in axialer, koronarer und sagittaler Richtung. Gegenwärtig findet zu Forschungszwecken die Anwendung von MRT-Geräten mit einer Feldstärke von 7 T, 9,4 T, 11,7 T und $21 \mathrm{~T}$ statt.
In der klinischen Routine findet die MRT-Diagnostik heute zahlreiche Anwendungen. Verschiedenste Verfahren und Systeme wurden entwickelt, welche die Anwendung und Nutzung der MRT heute zusätzlich verbessern. Hierzu zählen u.a. technische Erweiterungen wie die Perfusions-MRT, funktionelle MRT, MR-Elastografie, Echtzeit-MRT oder Magnetresonanzangiografie. Hinsichtlich der Bauform unterscheidet man offene und geschlossene MRT-Systeme. $\mathrm{Zu}$ den neuesten Anwendungsbereichen zählt u.a. die intraoperative MRT-Diagnostik.

Der Vorteil der MRT im Vergleich zu anderen radiologischen Untersuchungsverfahren liegt in der häufig besseren Darstellbarkeit bestimmter Organe und Strukturen aufgrund unterschiedlicher Signalintensitäten der Gewebe. $\mathrm{Zu}$ den deutlich besser erkennbaren Strukturen im Vergleich zur CT-Diagnostik zählen u.a. das Gehirn und die Nerven. Die zusätzliche Gabe eines Kontrastmittels (z.B. Gadopentetat-Dimeglumin, Magnevist ${ }^{\circledR}$, Bayer Healthcare, Leverkusen) kann die Darstellung von z.B. Tumorgewebe oder Entzündungsherden deutlich verbessern.

$\mathrm{Zu}$ den Nachteilen der MRT-Diagnostik zählt u.a. eine deutlich reduzierte Darstellung knöcherner Strukturen, sowie die ausgeprägte Artefaktbildung durch im Patienten befindliche metallische Strukturen.

Per se sind nach aktueller Studienlage keine wesentlichen Nebenwirkungen bei der herkömmlichen MRT-Diagnostik bekannt, lediglich in Einzelfällen kam es bei Patienten zu temporären Lichterscheinungen im Auge, sog. Phosphenen, sowie zu kurz andauernden Schwindelattacken.

$\mathrm{Zu}$ den neuesten Errungenschaften in der modernen MRT-Diagnostik zählt die Modifizierung und Anwendung spezieller präformierter Spulen, die eine deutlich verbesserte Darstellung bestimmter Zielstrukturen, wie z.B. der Brust, der Ohren oder der Kiefer ermöglichen. Diese Spulen verfügen i.d.R. über flexible Gelenke und ermöglichen somit, durch das direkte Anlegen der Spule an die zu untersuchende Struktur, eine deutlich erhöhte Auflösung der Bildqualität.

In einzelnen medizinischen Zentren findet seit einigen Jahren zudem die Anwendung von intraoperativen MRT-Ge- 

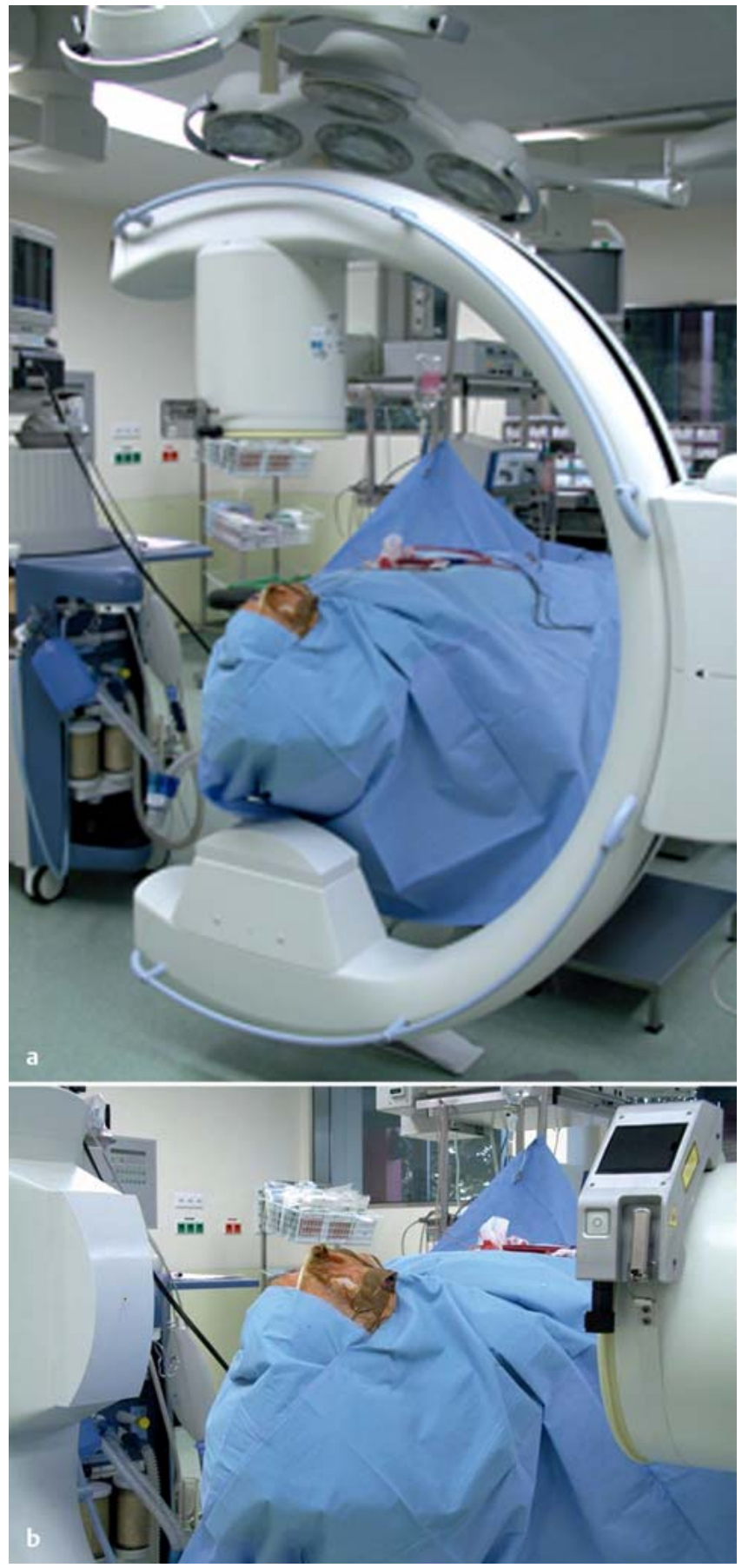

Abb. $4 \mathbf{a}$ und $\mathbf{b}$

Die Anwendung eines 3-D-C-Bogensystems (ARCADIS Orbic 3D, Siemens, Erlangen) zur intraoperativen Darstellung des Gesichtsschädels.

\section{3-D-C-Bogen (DVT; digitale Volumentomografie)}

Die digitale Volumentomografie hat innerhalb der letzten Dekade in der Mund-, Kiefer- und Gesichtschirurgie Einzug in die Operationssäle gehalten $[5,6]$. Im Gegensatz zur CT-Technologie nutzt die digitale Volumentomografie ein konisches Strahlenbündel, das durch einen großen Sensor aufgefangen wird. Aus diesem wesentlichen technischen Unterschied leitet sich auch das im angloamerikanischen Sprachraum gebräuchliche Akronym CBCT (Cone-Beam Computed Tomography) ab. Das DVT wird bei den gängigen Systemen in einen isozentrischen C-Bogen integriert, der bei Aufnahme $190^{\circ}$ um das zu untersuchende Gebiet abfährt. Nachteile dieser Technologie liegen in der Anwendung von ionisierender Strahlung, der ausschließlichen Beurteilbarkeit von Hartgewebe in den Aufnahmen, der eingeschränkten Bildqualität sowie des kleinen „field of view“, das mit einer Aufnahme erfasst werden kann (s. Abb. 2 und 3). Vorteile bestehen in der Erzeugung von 3-dimensionalen Bildserien mit einer guten Darstellung der Hartgewebe innerhalb kürzester Zeit intraoperativ und dies mit einer deutlich geringeren Strahlenbelastung als eine vergleichbare konventionelle CT-Aufnahme. Zudem ist das Gerät mobil und vergleichsweise kompakt, sodass es in großen Operationszentren in allen Sälen eingesetzt werden kann und nicht an einen Standpunkt gebunden ist. Abb. 4a und b zeigt ein 3-D-C-Bogensystem im Einsatz.

3-D-C-Bogensysteme liefern 3-dimensionale Bilder und erlauben Aussagen über die Hartgewebe. Sie sind mobil und im Vergleich relativ kostengünstig. Die Bildqualität ist CT-Aufnahmen nicht ebenbürtig, allerdings wird der Patient einer geringeren Strahlenbelastung ausgesetzt.

\section{Intraoperative Navigationssysteme}

Die Nutzung von intraoperativen Navigationssystemen im Bereich der Mund-, Kiefer- und Gesichtschirurgie ist bei Tumorresektionen im Bereich des Mittelgesichts, der Augenhöhlen und der Schädelbasis, der sekundären Rekonstruktion größerer Defekte und bei der primären Versorgung ausgedehnter Mittelgesichts- und Orbitafrakturen etabliert. Diesen Indikationsfeldern ist eine komplexe 3-dimensionale Struktur zu eigen,
Die Anwendung der MRT-Diagnostik in solchen Hybrid-OPs findet aktuell vorwiegend auf den Gebieten der Neurochi-
Intraoperative MRT-Systeme liefern hochauflösende 3-dimensionale Bilder vorwiegend der Weichteile. Vorteilhaft ist, dass die Patienten keiner Strahlenbelastung ausgesetzt sind. Nachteilig erscheinen die hohen Kosten sowie ein schwieriges intraoperatives Handling (kein Metall in der Nähe des MRT-Systems). 


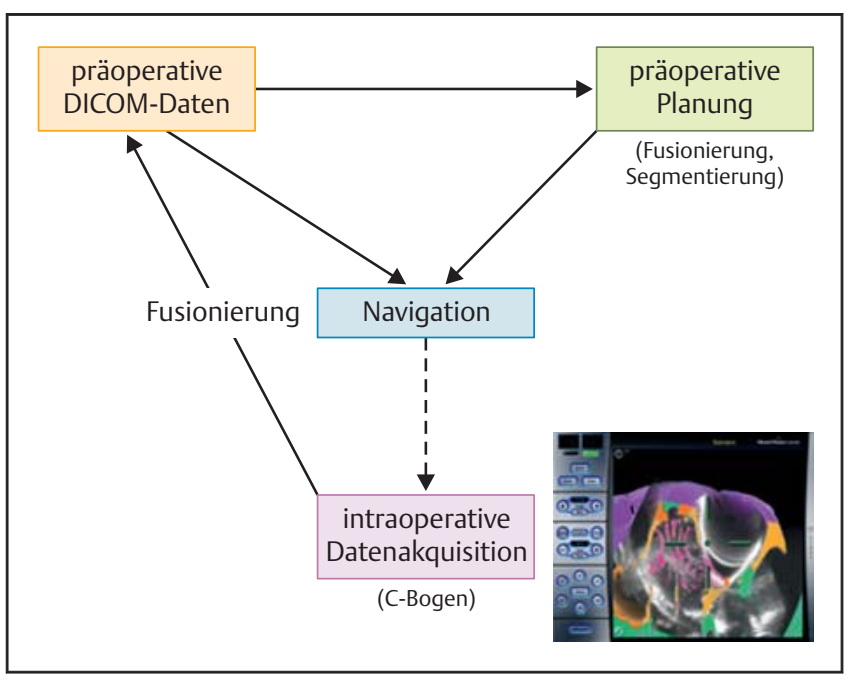

Abb. 5 Workflow zur Vorbereitung der intraoperativen Navigation. Basis sind präoperative DICOMDaten, die nach Laden in die Planungssoftware bearbeitet und markiert werden können. Zusätzlich können intraoperativ aufgenommene Bilddaten mit den präoperativen Daten fusioniert werden, um die Daten der Realsituation anzupassen.
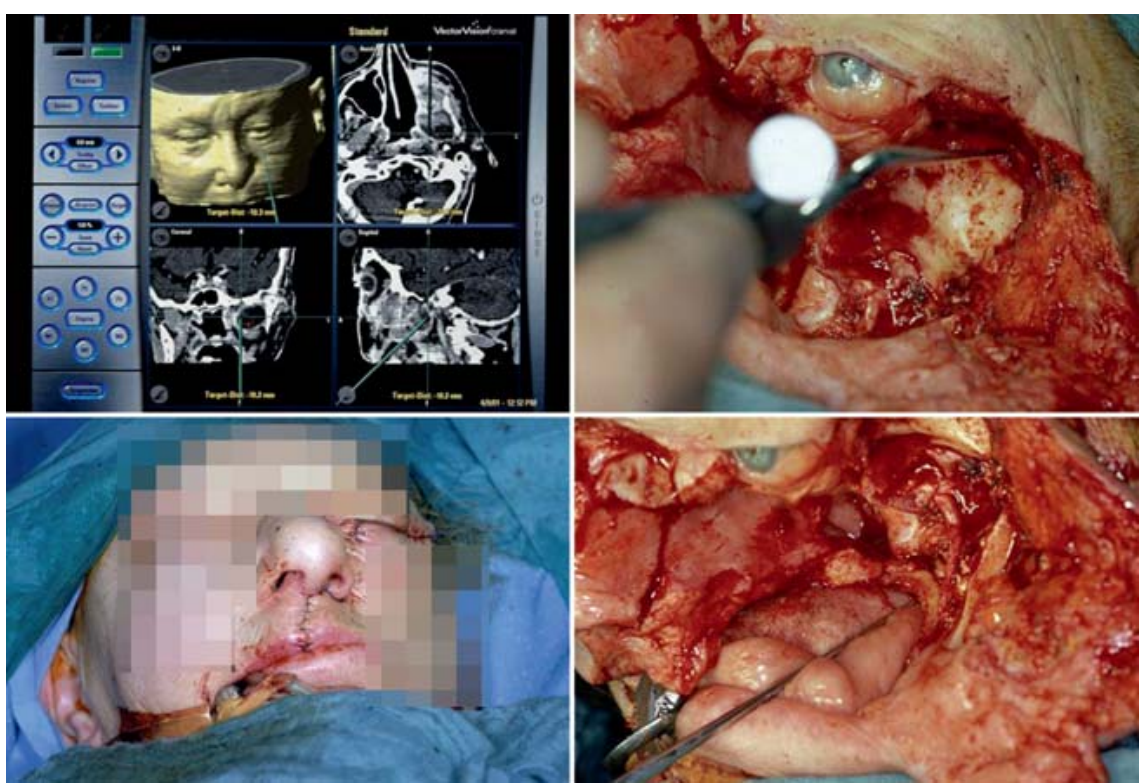

Abb. 6 Navigationsgestützte Tumorresektion bei schädelbasisnaher Lokalisation eines rezidivierten Ameloblastoms. Hier wird die Diskrepanz zwischen der auf präoperativen Bilddaten basierenden Darstellung des Navigationssystems und dem intraoperativen Situs besonders deutlich.

sodass das Navigationssystem den Operateur bei der Wiederherstellung der korrekten Struktur in Form und Größe unterstützt. Darüber hinaus kann das Auffinden von Fremdkörpern vereinfacht und so die Operationszeiten verringert werden.

Die auf dem Markt befindlichen Systeme (u.a. Kick ${ }^{\circledR}$, Brainlab AG, Feldkirchen, Deutschland, oder Fusion Navigation System $^{\circledR}$, Medtronic International, Tolochenaz, Schweiz) nutzen entweder eingespielte präoperativ vorbereitete Daten von 3-dimensionalen Schnittbildern (CT, DVT oder MRT) oder intraoperativ akquirierte Daten, um diese auf einem Monitor intraoperativ mit simultaner Anzeige eines chirurgischen Instruments wiederzugeben (Abb. 5). Dabei wird die Position des Instruments in Echtzeit in den Schnittbildern wiedergegeben, sodass der Operateur jederzeit über die genaue Position des Instruments informiert ist. Nach Einspielen der Daten präoperativ in die Planungssoftware des Navigationssystems kann die Markierung der operationsrelevanten Strukturen präoperativ erfolgen.

Ein Hauptproblem der Navigationssysteme besteht im zunehmenden Verlust der Genauigkeit im Verlauf der Operation. Bedingt durch die chirurgische Intervention kommt es unweigerlich zu einer Veränderung und einem zunehmenden
Abweichen der in realo bestehenden Situation von der im Gerät gespeicherten, präoperativen Situation (Abb.6). Dies kann im Zweifelsfall bis zum Verlust der Navigationsfähigkeit führen. Um diese Abweichung wieder auszugleichen, können intraoperative Bilddaten mit den im System eingespielten präoperativen Daten fusioniert werden.

Die intraoperative Bildgebung ist für $\mathrm{Na}$ vigationssysteme von großer Bedeutung, da die Genauigkeit der Navigationsdaten im Verlauf der Operation abnimmt. Dieser Effekt kann durch das Aufspielen von intraoperativ erzeugten 3-dimensionalen Bildern wieder ausgeglichen werden.

\section{Die intraoperative Bildgebung im klinischen Alltag}

Aufgrund der Mobilität und der leichten und schnellen Anwendbarkeit hat sich in unserer Klinik die reguläre Nutzung eines 3-D-C-Bogensystems etabliert. Nach Reposition und Osteosynthese einer Gesichtschädelfraktur erfolgt eine intraoperative Stellungskontrolle, um ggf. sofort eine Revision durchführen zu können. Der Zeitverlust durch die Bildgebung ist dabei als nicht relevant im Hinblick auf den Nutzen zu bezeichnen. Ein Revisionsbedarf tritt dabei bei ca. $10 \%$ der Fälle auf, wobei eine sofortige Revision erfolgen kann [7]. Als vielversprechende Erweiterung des Spektrums ist die Möglichkeit zu nennen, nach stabiler geschlossener Reposition von Mittelgesichtsfrakturen und intraoperativer Röntgenkontrolle auf eine offene Darstellung und Osteosynthese zu verzichten [6].

Interessant ist aus unserer Sicht aber die Perspektive mobiler CT-Systeme, welche eine noch bessere Bildqualität und v.a. eine Weichgewebsdarstellung versprechen.

\section{Schlussfolgerungen}

Die intraoperative Bildgebung mit Sonografie, MRT, CT und 3-D-C-Bogensystemen ist eine wertvolle Bereicherung und wichtige Unterstützung für die Mund-, Kiefer- und Gesichtschirurgie und bietet wesentliche Vorteile im Hinblick auf eine sofortige Kontrolle des Operationserfolgs. Bei sorgfältiger Abwägung der Vor- und Nachteile der auf dem Markt befindlichen intraoperativen Systeme und dem bestehenden Bedarf kann ein Gerätetyp ausgewählt werden, 
mit dem sich die Durchführung der Aufnahmen problemlos in den Operationsablauf integrieren lässt.

\section{Literatur}

${ }^{1}$ Friedrich RE, Heiland M, Bartel-Friedrich S. Potentials of ultrasound in the diagnosis of midfacial fractures*. Clin Oral Investig 2003; 7: 226-229

2 Stieve M, Issing PR, Mack KF et al. [Indications of intraoperative ultrasound in head and neck surgery]. Laryngorhinootologie 2012; 91: 422-426

3 Novelli G, Tonellini G, Mazzoleni F et al. Surgical navigation recording systems in orbitozygomatic traumatology. J Craniofac Surg 2012; 23: 890-892
${ }^{4}$ Jolesz FA. Future perspectives for intraoperative MRI. Neurosurg Clin N Am 2005; 16: 201-213

${ }^{5}$ Heiland M, Schulze D, Blake F et al. Intraoperative imaging of zygomaticomaxillary complex fractures using a 3D C-arm system. Int J Oral Maxillofac Surg 2005; 34: 369-375

${ }^{6}$ Wilde F, Lorenz K, Ebner AK et al. Intraoperative imaging with a 3D C-arm system after zygomatico-orbital complex fracture reduction. J Oral Maxillofac Surg 2013; 71: 894910

7 Klatt JC, Heiland M, Blessmann M et al. Clinical indication for intraoperative 3D imaging during open reduction of fractures of the neck and head of the mandibular condyle. J Craniomaxillofac Surg 2011; 39: 244-248
Dr. med. Dr. med. dent.

Henning Hanken

Wissenschaftlicher Mitarbeiter

Dr. med. Christian Lohse

Wissenschaftlicher Mitarbeiter

Dr. med. Dr. med. dent.

Alexandre Thomas Assaf

Wissenschaftlicher Mitarbeiter

Prof. Dr. med. Dr. med. dent.

Max Heiland

Direktor

Klinik und Poliklinik für Mund-, Kiefer- und Gesichtschirurgie Universitätsklinikum HamburgEppendorf

Martinistr. 52

20246 Hamburg

m.heiland@uke.de 